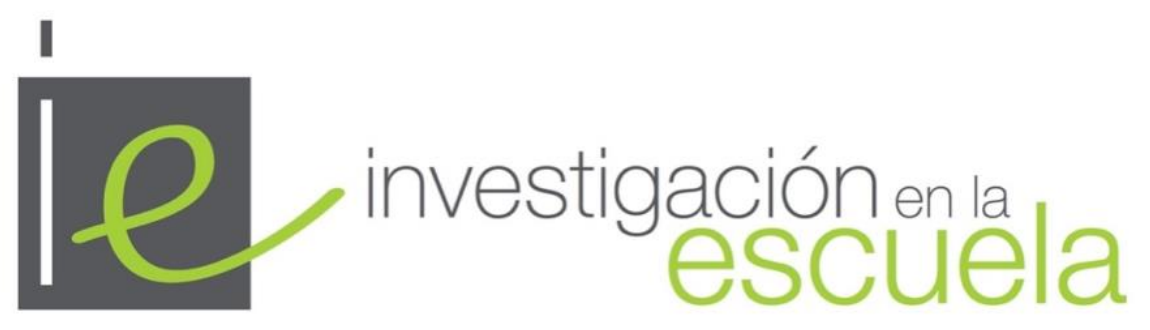

Revista de Investigación e Innovación Educativa nº 105, 2021 | e-ISSN 2443-9991

\title{
E1 prácticum RIECU a evaluación. De la innovación a la investigación educativa como herramienta para transformar la práctica
}

\section{Evaluating the Riecu Practicum. From innovation to educational research as a strategy for practice improvement}

Dra. Rosario Mérida-Serrano, es Catedrática en el Departamento de Educación de la Facultad de Ciencias de la Educación en la Universidad de Córdoba (España)·ed1meser@uco.es · Orcid https://orcid.org/0000-0001-93893263

Dra. $\mathbf{M}^{\mathbf{a}}$ Elena González-Alfaya, es Profesora Contratada Doctora en el Departamento de Educación de la

iD Facultad de Ciencias de la Educación en la Universidad de Córdoba (España) · ed2goalm@uco.es · Orcid https://orcid.org/0000-0001-8264-3680

Dra. $\mathbf{M}^{\mathrm{a}}$ de los Ángeles Olivares-García, es Profesora Contratada Doctora en el Departamento de Educación de la Facultad de Ciencias de la Educación en la Universidad de Córdoba (España)·ed1olgam@uco.es · Orcid https://orcid.org/0000-0002-9440-5628

D. Miguel Muñoz-Moya, es Profesor Sustituto Interino en el Departamento de Educación de la Facultad de

(iD) Ciencias de la Educación en la Universidad de Córdoba (España) · p62mumom@uco.es · Orcid https://orcid.org/0000-0001-6250-2995

Dra. Julia Rodríguez-Carrillo, es Contratada Predoctoral en el Departamento de Educación de la Facultad de Ciencias de la Educación en la Universidad de Córdoba (España) · m12rocaj@uco.es · Orcid https://orcid.org/0000-0003-0725-3054

Cómo citar este artículo

Mérida-Serrano, R., González-Alfaya, E, Olivares-García, M.A., Muñoz-Moya, M. y Rodríguez-Carrillo, J. (2021). El prácticum RIECU a evaluación. De la innovación a la investigación educativa como herramienta para transformar la práctica. Investigación en la Escuela, 105, 11-24. doi: https://doi.org/10.12795/IE.2021.i105.02

Resumen. Este trabajo tiene como objetivo analizar el impacto que ha tenido en el estudiantado su participación en una experiencia de innovación educativa, vinculada al Prácticum III de cuarto curso del Grado de Infantil de la Facultad de Ciencias de la Educación de la Universidad de Córdoba. Se incluyen cuatro elementos innovadores que diferencian a este prácticum del ordinario: (1) Selección, mediante criterios de excelencia, de las maestras y los maestros que asumen la tutoría profesional; (2) Configuración de la pareja educativa compuesta por el o la estudiante universitaria y la tutoría profesional; (3) Documentación pedagógica; y (4) Desarrollo de procesos de investigación-acción colaborativos. Concretamente, se analizan la adquisición de competencias profesionales, las fortalezas y debilidades expresadas por los y las estudiantes, las tutorías académicas y profesionales, así como la función desempeñada por las asesoras del Centro de Profesorado vinculadas a esta innovación. Esta mirada poliédrica facilita un acuerdo interjueces y una triangulación de los datos recabados en este estudio. Se utiliza una metodología de investigación evaluativa que se apoya en dos instrumentos de recogida de información: un grupo focal e informes reflexivos del alumnado de prácticas. Mediante la técnica de análisis del discurso, los datos revelan que el estudiantado incrementa sus competencias docentes relacionadas con la observación sistemática del aula, la actitud de escucha a la infancia, el autocontrol emocional y la reflexión sobre su práctica.

Abstract. The present paper is aimed at analysing the impact derived from student teachers participating in an 
educational innovation experienced, linked to the course Practicum III of the fourth year of the Early Childhood Education (ECE) Degree at the Faculty of Education in the University of Córdoba. Four innovative elements are considered, which differentiate this practicum from the ordinary one: (1) the selection, following excellence criteria, of ECE teachers who exert the role of professional tutors; (2) the forming of the educational pair, integrated by a student teacher and an ECE teacher; (3) pedagogical documentation; and (4) the development of collaborative action-research processes. More precisely, several aspects are analysed: the acquisition of teaching competences, strengths and weaknesses of the innovation experience as expressed by the student teachers, as well as the role exerted by the professional tutors and the advisors from the Teacher Training Centre who are also part of this innovation. This polyhedric perspective helps in gaining inter-judge agreement and the triangulation of data. An evaluative method is followed which relies in two collection techniques: a focus group and student teachers' reflective diaries. By means of discourse analysis, data reveal that, after participating in this innovative practicum, prospective teachers improve their professional competences, specifically those that have to do with the systematic observation of the classroom, listening to children, emotional self-control, and reflection about their practice.

\section{Palabras clave $\cdot$ Keywords}

Práctica pedagógica, formación de profesorado, documentación pedagógica, innovación pedagógica, investigaciónacción, investigación educativa.

Teaching practice, teacher education, pedagogical documentation, educational innovation, action research, educational research.

\section{Introducción}

Multitud de innovaciones, desarrolladas en los diferentes niveles del sistema educativo, ponen de manifiesto los beneficios que se derivan del trabajo por competencias (Bellver, 2016; Rodríguez y Cruz, 2020; Villela y Torres, 2015), del abordaje interdisciplinar de los contenidos (Pozuelos-Estrada et. al, 2012), del aprendizaje cooperativo (Mena et al., 2017), de la introducción de la educación democrática en los procesos de formación (Zeichner et al., 2015), de la incorporación de la educación en entornos naturales (Aragón y Jiménez-Tenorio, 2015; Charles, 2009) o de las metodologías activas de aprendizaje (Shi et. al, 2019). Todos estos estudios dan cuenta de la búsqueda permanente de mejora del sistema educativo que persiguen los y las profesionales comprometidos con el aprendizaje y la formación integral de su alumnado. Un deseo de mejora que pretende superar las debilidades que se identifican en informes internacionales como PISA 2018 (2019), en el que se muestra el fracaso y abandono escolar temprano, la enseñanza excesivamente memorística y poco centrada en las competencias para resolver problemas vitales y la falta de equidad en los rendimientos escolares, como principales amenazas para el logro de una sociedad del conocimiento sostenible y justa.

Ante esta persistente realidad, la innovación educativa y la renovación pedagógica siguen siendo necesarias porque urge transformar una realidad escolar en la que sigue prevaleciendo el uso hegemónico del libro de texto, como forma de reproducir el conocimiento, una enseñanza uniformante y una metodología transmisiva que, en más ocasiones de las deseables, desatiende la creatividad, la diversidad y el talento de cada discente (Alsina y Batllori, 2015).

Pero mejorar la calidad de las situaciones educativas que se plantean en las aulas de Educación Infantil y Primaria requiere, necesariamente, incrementar la calidad en la formación de sus docentes. Los informes de distintos organismos internacionales como la OECD (2006), UNICEF (2017) con el informe relativo al Estado Mundial de la Infancia. Niños en un mundo digital, la European Early Childhood Education Research Association (Johansson \& Einarsdottir, 2017) y su documento sobre la ciudadanía del mañana, la National Association for the Education of Young Children (NAEYC, 2002), y su informe sobre estándares de aprendizaje en Educación Infantil, o el informe TALIS 2018 (2019), refrendan que la calidad del profesorado es la variable crítica que más influye en el aprendizaje del alumnado.

Por esta razón, nuestra mirada se centra en analizar la calidad de la formación que estamos ofreciendo desde la universidad a los y las futuras docentes de Educación Infantil. ¿Estamos proporcionando al estudiantado las competencias profesionales necesarias para la escuela del siglo XXI? ¿Desarrollamos una formación excesivamente técnica y desatendemos el trabajo del pensamiento crítico y transformador? ¿Son las prácticas en la escuela momentos para la integración de la teoría y la práctica en un modelo docente innovador, o, por el contrario, los futuros docentes se forman por reproducción de modelos docentes tecnocráticos? Estos y otros muchos interrogantes son el germen del que surge la Red de Infantil-EscuelaCentro de Formación del Profesorado-Universidad (en adelante, RIECU) (Mérida-Serrano, 2005; OlivaresGarcía et al., 2016; Rodríguez-Carrillo et al., 2019). 


\subsection{RIECU, una plataforma de innovación para la mejora de la formación de los y las} futuras docentes de Infantil

RIECU surge en el año 2007/2008 y sigue funcionando hasta la actualidad, a raíz del trabajo colaborativo de docentes que, perteneciendo a escuelas infantiles, al Centro de Formación del Profesorado (en adelante, CEP) y a la universidad comparten una actitud crítica ante la formación inicial universitaria (Pérez Gómez, 2012), una concepción de infancia como co-constructora de cultura (Moss et al., 2005) y una concepción socioconstructivista (Mauri et al., 2017; Ribosa, 2020) e inclusiva del aprendizaje (Arbouet, 2010).

RIECU se constituye como una red profesional colaborativa que actúa como ecosistema innovador en la formación inicial del estudiantado universitario del Grado de Educación Infantil para conseguir incrementar el dominio de las competencias profesionales de los futuros docentes, al aproximarlos a la metodología de Aprendizaje Basado en Proyectos (en adelante, ABP).

Para ello, RIECU diseña un prácticum innovador, al considerar que se trata de un periodo formativo de máxima fertilidad para la adquisición de competencias profesionales docentes, en el que se integran de manera experiencial la teoría y la práctica e interaccionan profesionales involucrados en la formación inicial y docentes en ejercicio. Es decir, como indica Pérez Gómez (2012), se teoriza la práctica y se practica la teoría. En definitiva, el prácticum es un momento en el que el pensamiento práctico del docente se pone a prueba, interaccionando sus concepciones teóricas con sus creencias implícitas, las cuales no son conscientes y están vinculadas a los afectos, emociones, expectativas, valores y necesidades, resultando muy resistentes al cambio. Se necesita que el estudiantado universitario tome conciencia, en la práctica, de la disonancia entre sus teorías declaradas (Korthagen, 2010) -a menudo expuestas en los exámenes mediante un discurso racional, fruto de la memorización de asignaturas atomizadas y alejadas de la realidad de las aulas- y sus teorías en uso -sus comportamientos profesionales en el aula cuando interactúa con el alumnado de Educación Infantil, diseña situaciones de aprendizaje y se relaciona con las familias-. Como señala Pérez Gómez (2010, p. 47) "los esquemas intuitivos e inconscientes de comprensión y de actuación solamente se forman y reconstruyen mediante las experiencias prácticas en los contextos reales".

Pero no cualquier situación educativa estimula la transformación del pensamiento práctico docente en la dirección deseada. Si ofrecemos al estudiantado universitario modelos transmisivos, reproductivos y rutinarios, probablemente, afianzarán sus creencias implícitas previas, al identificarse con maestros y maestras legitimadas por el sistema educativo, que devalúan los marcos teóricos más actualizados, al valorarlos como utópicos y poco útiles para resolver los desafíos de la práctica.

¿Qué características ha de tener, entonces, un prácticum para impulsar la transformación del pensamiento práctico? En la red RIECU, tras más de una década de innovación e investigación educativa, se identifican cuatro elementos de innovación:

(1) Selección, mediante criterios de excelencia, de las maestras y los maestros que asumen la tutoría profesional. Desde el CEP se diseña un curso formativo sobre el Prácticum RIECU, al que pueden inscribirse de manera voluntaria solo docentes que tengan una formación acreditada previa sobre ABP y una experiencia de aplicación práctica de esta metodología en sus aulas.

(2) Configuración de la pareja educativa, compuesta por el o la estudiante universitaria y la tutoría profesional. Se trata de una relación profesional, centrada en la horizontalidad y colaboración, que favorece la autonomía profesional del aprendiz de docente.

(3) Documentación pedagógica. Realizada por el alumnado de Ciencias de la Educación sobre las situaciones de enseñanza-aprendizaje y sobre las interacciones del alumnado de Educación Infantil, así como de la participación de sus familias. Se utiliza como herramienta para reflexionar sobre su práctica en las sesiones de investigación-acción.

(4) Desarrollo de procesos de investigación-acción colaborativos (en, al menos, una sesión semanal) realizados por el estudiantado, junto a la tutoría profesional, la tutoría académica y asesoras de Educación Infantil del CEP.

A continuación, vemos con más detenimiento cada uno de estos elementos innovadores.

\subsection{Maestras comprometidas con la calidad y la formación continua, como modelos profesionales excelentes}

La socialización inicial de los futuros docentes, de la mano de maestras con experiencia, produce un alto impacto en la configuración de su incipiente identidad profesional (Marín-Cano et al., 2019). Se produce un fuerte aprendizaje por modelado del estudiantado universitario, al sumergirse en contextos prácticos 
liderados por docentes que gozan de una profesionalidad acreditada institucionalmente. Su inexperiencia, su inseguridad y la carencia, en la mayoría de los casos, de experiencias previas como docentes, colocan al profesorado en formación en una situación de dependencia y vulnerabilidad respecto a las docentes en ejercicio (Olivares-García et al., 2016).

En estos primeros estadios de su desarrollo profesional no disponen de competencias ni esquemas críticos, suficientemente sólidos, que les permitan cuestionar prácticas educativas tecnocráticas o reproductivas (Zeichner et al., 2015). Por el contrario, se produce un proceso de inmersión acrítico cuyo objetivo prioritario es desenvolverse eficazmente en el aula, en muchas ocasiones siguiendo la estela y los patrones de comportamiento de las docentes, sin repensar si las situaciones de aprendizaje creadas, su interacción con el alumnado o los principios de intervención educativa son los más adecuados.

Desarrollan, en la mayoría de los casos, una conducta profesional adaptativa y mimética que sintoniza con el modelo educativo que prevalece en el aula, relegando a un segundo o tercer plano las directrices teóricas, dado que, a veces, la distancia entre la ardua teoría -vinculada al ámbito cognitivo y racional- y la seductora práctica -relacionada con componentes emocionales y afectivos- es insalvable. Esta perversa disonancia entre teoría y práctica impide que el estudiantado universitario, pese a tener todas sus asignaturas aprobadas, pueda identificar prácticas en las aulas infantiles poco coherentes con una teoría del aprendizaje socioconstructivista, un modelo docente reflexivo o una metodología centrada en los niños y niñas. Por el contrario, se comienza a legitimar, por parte del estudiantado en formación, un discurso docente que desvaloriza la aportación teórica como una 'moda pedagógica' pasajera y ajena a las verdaderas necesidades que se derivan de la práctica, expresando a la vuelta de los colegios de prácticas que lo que han aprendido es que la teoría no sirve para casi nada (Mérida-Serrano et al., 2019).

Para revertir este divorcio entre teoría y práctica hemos seleccionado en el prácticum RIECU a maestras que, además de tener una excelente e innovadora práctica de aula, mantienen un compromiso con la formación permanente, lo cual les permite manejar los conceptos teóricos que respaldan sus actuaciones en el aula. No solo deben saber actuar eficazmente en el aula, sino que deben saber justificar por qué lo hacen, evolucionando de un mero estadio profesional intuitivo, caracterizado por el ensayo-error, a una actitud profesional fundamentada en una teoría que se reformula y evoluciona en su interacción permanente con la práctica.

En resumen, no cualquier maestra se considera válida para formar a las futuras generaciones docentes y es necesario seleccionar a aquellas profesionales que ofrecen un referente profesional digno de ser imitado.

\subsection{La pareja educativa. Construyendo una relación dialógica entre docentes de hoy y del mañana}

Existe abundante literatura científica en relación a las bondades de la pareja educativa como estrategia de profesionalización y herramienta para incrementar la calidad educativa de las aulas infantiles, mostrándose como uno de los pilares fundamentales del modelo reggiano (Caruso, 2013; Coll y Moll, 2005; Hoyuelos, 2004; Luna Arcos y Elorza Ibáñez de Gauna, 2009).

La concepción de pareja educativa que se trabaja en RIECU se sustenta en aportaciones como la de Cabrera (1988) (citado en Civarolo y Pérez Andrada, 2013), quien señala que:

Dos docentes significan dos lenguajes, dos estrategias, tal vez, dos metodologías. Dos relaciones posiblemente distintas. Dos diálogos, dos enfoques, dos estímulos, dos apreciaciones de procedencia desigual. Dos presencias que permitirán al estudiante ganar en vivencias que le ensancharán el mundo. Su mundo. [...] En síntesis, una lengua más rica, más libre, más suelta, más plena, al servicio de los estudiantes (p.17).

Las ventajas de la pareja educativa son innumerables: (1) Incentiva la colaboración entre docentes. Es necesario que en el marco de una escuela colaborativa los procesos de cooperación comiencen por las propias docentes; (2) Propicia un diálogo profesional no jerárquico, una relación donde se puede discutir y mostrar de forma pública los diferentes puntos de vista; (3) Facilita un entorno emocional de apoyo, donde es más fácil desarrollarse profesionalmente —en la cotidianidad—, al compartir las emociones y las ideas que supone la extraordinaria y compleja tarea de educar; y (4) Hace posible la complementariedad de miradas, la observación compartida, como ejercicio de heteroevaluación, donde el intercambio de puntos de vista se torna en un requisito imprescindible al reconocer que un individuo aislado no es poseedor de la verdad. Se trata de asumir, humildemente, que somos «incompletos» y que existe la necesidad de encontrarnos con los demás para poder crecer y mejorar personal y profesionalmente.

La concepción de pareja educativa, que tras años de innovación e investigación educativa (MéridaSerrano et al., 2020), suscribimos en RIECU es: 
Un par de personas que poseen conocimientos docentes, cuyo nivel de desarrollo profesional y experiencia es diferente, pero que comparten una concepción de infancia, de educación y de escuela similar, lo cual propicia una coordinación eficaz e intensa al compartir un mismo escenario educativo. Generalmente se comparten las acciones en el aula de manera equitativa, estableciéndose un diálogo simétrico e igualitario entre ambas (p. 28).

La configuración de las parejas educativas en RIECU se realiza de forma aleatoria entre las maestras y las estudiantes del Grado de Educación Infantil. Se les ofrece un espacio de encuentro previo a la intervención en el aula, dedicado a la clarificación de roles, a la planificación de las actuaciones, a la fijación de criterios de coordinación con las familias y a establecer las pautas de interacción con el alumnado. Tras esta sesión inicial, las parejas educativas de RIECU comienzan su interacción en las aulas infantiles, que durará dos meses, y que dará lugar al establecimiento de diferentes vínculos profesionales y, en algunos casos, personales. Esta relación dependerá de la idiosincrasia de cada una de las personas de la pareja y, sobre todo, de su compatibilidad docente y del vínculo comunicativo que se cree entre ambas.

\subsection{La documentación pedagógica. Una herramienta para reconstruir el discurso narrativo sobre la infancia}

En una de las revisiones más exhaustivas publicadas recientemente por Alaçam \& Olgan (2021) sobre la documentación pedagógica en Educación Infantil, se analizan 24 estudios empíricos, en los que se señalan sus posibles beneficios: (1) Como herramienta de observación sistemática en el aula; (2) Como estrategia para hacer visible el aprendizaje del alumnado (Rintakorpi \& Reunamo, 2017); (3) Como técnica para fomentar la participación del alumnado en la gestión democrática del aula (Sousa, 2019); (4) Como estrategia para favorecer las habilidades sociales y metacognitivas del alumnado (Aras \& Tantekin Erden, 2019) y (5) Como vehículo de comunicación con las familias.

Desde RIECU se asume la concepción que plantea Hoyuelos (2004), al considerar que la documentación pedagógica responde a un plan detallado de observación y escucha de la infancia, cuya finalidad es entender la cultura infantil: su forma de pensar, hacer, preguntar, teorizar o desear. Escuchar significa estar atento, con todos los sentidos, a reconocer todos los lenguajes de la infancia en su relación con el mundo. Escuchar es una actitud que nos debe remover desde dentro para vaciarnos de prejuicios. Es romper para construir.

La documentación consiste en la recogida y exposición sistemática y estética (a través de escritos, imágenes, paneles, vídeos, palabras de los niños y las niñas, productos gráficos...) de los procesos educativos. No se trata solo de plasmar lo acontecido, sino de construir -en diversos formatos- un producto público que dé cuenta narrada de lo vivido. La documentación, de forma intrínseca, incorpora una acción selectiva de elegir lo relevante, lo significativo, lo sorprendente e inesperado, lo que hace diferente cada día en una escuela infantil. Es necesario ajustar el foco para seleccionar, enfocar y encuadrar la mirada en aquellos aspectos que puedan ser reconstruidos para ofrecer una visión interesante y comunicativa de las experiencias vividas.

Por ello, en el caso de RIECU, la documentación pedagógica se utiliza, fundamentalmente, como estrategia para mejorar las competencias profesionales del estudiantado del Grado de Educación Infantil, concretamente su capacidad de observación sistemática y su capacidad para reflexionar en y sobre su práctica.

\subsection{Los procesos de investigación-acción colaborativos. Reflexionar para mejorar}

Existen multitud de trabajos que resaltan la idoneidad de la investigación-acción para la formación del profesorado (Aranguren Peraza, 2007; Aras, 2021; Carr y Kemmis, 1988; Contreras Domingo, 1994; Elliott, 1993; Kemmis y McTaggart, 1992; McNiff, 2002). Sus aportaciones se vinculan a los procesos de reflexión en y sobre la práctica que facilitan, la conexión práctica-teoría que permiten, el trabajo colaborativo que inspiran, así como el desarrollo de competencias profesionales que propician.

La red RIECU opta por el concepto de investigación-acción colaborativa que nos proponen Boggino y Rosekrans (2007), al concebirla como un camino que facilita el cambio e innovación de la práctica educativa, porque desarrolla un proceso de reflexión crítica y sistemática sobre la propia acción educativa en compañía de otras miradas.

Los procesos de investigación-acción en el prácticum RIECU siguen las fases propias de esta metodología, abordando de forma circular tres momentos: (1) Planificación; (2) Acción y (3) Reflexión. Se van acometiendo estas fases de la siguiente manera: 


\subsection{Fases del proyecto de innovación del Prácticum RIECU}

Las fases en las que se articula el prácticum innovador RIECU son:

(1) Planificación. Se reúne el Equipo de Coordinación de RIECU —integrado por docentes de Educación Infantil, profesorado del Grado de Educación Infantil y asesoría de Educación Infantil del CEP - para diseñar el cronograma de actuación del curso, seleccionar los materiales que se les aportará a las estudiantes de Grado, establecer los criterios de selección de las maestras y organizar la primera sesión del proyecto. En esta sesión inicial se produce el encuentro y se comienza el diálogo entre las maestras, las estudiantes, las asesoras y las profesoras universitarias.

(2) Formación inicial en la universidad y continua en el CEP. En esta fase se seleccionan a las maestras, siguiendo los criterios decididos en la fase anterior, y a las alumnas del Grado de Educación Infantil que, voluntariamente, deseen participar. En el CEP se celebra un curso de formación para las maestras participantes, incidiendo en la metodología de ABP y en el rol docente como profesional reflexivo en y sobre su práctica. Simultáneamente, en las aulas universitarias, en el marco de la asignatura de Estrategias de Intervención en Educación Infantil, se estudia la metodología de ABP incidiendo en sus fases, así como en los principios educativos que la sustentan.

(3) Interacción estudiantes-maestras. Se celebra un encuentro antes de las prácticas presenciales, en el que se definen las parejas educativas y se continúa el diálogo entre las maestras y las estudiantes. Se dan las directrices para la realización de los procesos de documentación pedagógica, así como de los procesos de investigación-acción que, semanalmente, deben desarrollar en una franja horaria sin atención directa a niños y niñas. Posteriormente, se inician las prácticas en los centros y, además de ir desarrollando sus respectivos proyectos, se celebran en los colegios 8 sesiones de carácter semanal (durante dos meses de prácticas) de reflexión colaborativa entre la maestra, la estudiante, la asesora y el profesor o la profesora universitaria. En esta fase las maestras RIECU también acuden a la Facultad de Ciencias de la Educación para participar en unos seminarios de análisis teoría-práctica sobre casos concretos extraídos de la práctica, relacionados con cuatro dimensiones: planificación didáctica, atención a la diversidad, convivencia y relaciones de la escuela con su entorno familiar y social. Se produce una socialización circular del conocimiento y las experiencias de todo el alumnado que participa en el proyecto de innovación.

(3) Evaluación. Una vez concluidas las prácticas presenciales se organiza un grupo focal con las maestras participantes, el profesorado universitario y las asesoras del CEP. Simultáneamente se analizan las valoraciones personales expresadas por las estudiantes universitarias en sus informes de prácticas, respecto a las fortalezas, debilidades y propuestas de mejora de esta innovación para su sostenibilidad futura.

\section{La investigación educativa como herramienta para evaluar el impacto de la innovación}

La red RIECU considera que cualquier proceso de innovación pedagógica debe ser adecuadamente evaluado para dilucidar su potencial formativo y, por ende, su pertinencia para la mejora educativa. Con este fin, RIECU utiliza la investigación educativa como una herramienta para evaluar el impacto que ha producido la innovación en sus protagonistas. En este trabajo analizamos el impacto del prácticum RIECU en las estudiantes participantes.

\subsection{Contexto}

El prácticum RIECU se inscribe en una línea de innovación educativa consolidada, que es financiada por la Universidad de Córdoba a través del Plan Propio de Innovación y Buenas Prácticas Docentes. Se trata de una convocatoria en concurrencia competitiva que se enmarca en la modalidad de colaboración escuela-centros educativos. Participa el alumnado del Prácticum III de cuarto curso del Grado de Educación Infantil, tras haber realizado el Prácticum I y II. Se trata de un prácticum de intervención, que incluye seminarios de preparación antes de las prácticas, las prácticas presenciales en los centros — dos meses-, seminarios de análisis durante las prácticas, una sesión de innovación y buenas prácticas y, para concluir, la presentación de un informe reflexivo de la experiencia.

\subsection{Participantes}

Durante el curso académico 2020/2021, fruto de la crisis sanitaria derivada de la pandemia y de las restricciones en la universidad y en los centros educativos, ha decrecido la participación, cifrándose en 249 las personas implicadas, distribuidas del siguiente modo: 
Tabla 1

Participantes

\begin{tabular}{c|c|c|c|c|c|c}
\hline $\begin{array}{c}\text { Maestras de } \\
\text { Infantil }\end{array}$ & \multicolumn{2}{|c|}{$\begin{array}{c}\text { Profesorado } \\
\text { universitario }\end{array}$} & $\begin{array}{c}\text { Asesoras del } \\
\text { CEP }\end{array}$ & $\begin{array}{c}\text { Estudiantes de } \\
\text { Grado de Infantil }\end{array}$ & \multicolumn{2}{c}{$\begin{array}{c}\text { Alumnado de 3 a } \\
\mathbf{6} \text { años }\end{array}$} \\
\hline 11 & $\begin{array}{c}\text { Mujeres } \\
4\end{array}$ & $\begin{array}{c}\text { Hombres } \\
1\end{array}$ & 2 & 11 mujeres & $\begin{array}{c}\text { Niñas } \\
116\end{array}$ & $\begin{array}{c}\text { Niños } \\
104\end{array}$ \\
\hline
\end{tabular}

TOTAL 249

\subsection{Interrogantes de investigación}

- ¿Qué competencias profesionales han adquirido las estudiantes al participar en el prácticum innovador RIECU?

- ¿Qué fortalezas han experimentado al participar en el prácticum innovador RIECU?

- ¿Qué debilidades y sugerencias proponen para mejorar el prácticum innovador RIECU?

\subsection{Metodología e instrumentos}

Se ha utilizado una metodología de investigación evaluativa, entendida "como investigación que implica un proceso riguroso, controlado y sistemático de recogida y análisis de información fiable y válida para tomar decisiones sobre un programa educativo" (Tejedor, 2000, p. 320).

\subsection{Procedimiento de análisis: instrumentos, codificación, categorización, y técnicas de análisis}

Se utilizan dos instrumentos para obtener una triangulación metodológica que apoye la credibilidad y veracidad de las conclusiones: (1) Grupo focal on-line (Onwuegbuzie et. al, 2011) (en el que participan 11 maestras, 2 asesoras y 2 profesoras universitarias) y se utiliza la plataforma Mentimeter como panel para compartir ideas, y (2) Informes reflexivos de prácticas de 11 estudiantes. Se utiliza la técnica de análisis del discurso, siguiendo un procedimiento mixto: deductivo -para establecer las dimensiones, como unidades de análisis macro que derivan de la teoría- e inductivo -para identificar las categorías que emergen del discurso de las personas participantes-. El discurso se codifica asignando la primera inicial según el colectivo al que pertenece: M, maestras; $\mathrm{A}$, asesoras; $\mathrm{P}$, profesoras y $\mathrm{E}$, estudiantes, seguido del número adscrito a cada participante y de la categoría que corresponda, de forma que, por ejemplo, el código M10,1.2. se refiere a la maestra a la que se ha identificado con el número 10 y cuyo discurso tiene que ver con la categoría 1.2. Para el análisis de datos se utiliza el programa Atlas.ti 9. El proceso de categorización responde a un acuerdo interjueces entre las dos profesoras universitarias que actúan como investigadoras, en el que se consensuan las categorías coincidentes y se eliminan las discrepantes. Los resultados del estudio se devuelven a todas las personas participantes antes de su difusión.

\section{Resultados}

Para mayor claridad analítica se organizan los datos en la siguiente matriz hermenéutica, que se corresponde con los interrogantes de investigación:

Tabla 2

Matriz. hermenéutica de las unidades de análisis

\begin{tabular}{l|l}
\hline \multicolumn{1}{c|}{ Dimensiones } & \multicolumn{1}{c}{ Categorías } \\
\hline \multirow{2}{*}{$\begin{array}{l}\text { 1. Competencias profesionales adquiridas } \\
\text { por el estudiantado universitario }\end{array}$} & 1.1. Observar sistemáticamente en el aula \\
\cline { 2 - 2 } & 1.2. Aprender a escuchar a la infancia \\
\cline { 2 - 2 } & 1.3. Control emocional para crear un buen clima de aula \\
\cline { 2 - 2 } & 1.4. Reflexionar sobre la práctica \\
\hline \multirow{2}{*}{ 2. Fortalezas } & 2.1. Inyección de moral \\
\cline { 2 - 2 } & 2.2. Seducción de la metodología ABP \\
\hline 3. Debilidades y sugerencias de mejora & 3.1. Limitaciones derivadas del COVID-19 \\
\hline
\end{tabular}




\subsection{Competencias profesionales adquiridas por el estudiantado universitario}

\section{Observar sistemáticamente en el aula}

Las maestras expresan que perciben una evolución en el dominio de las competencias profesionales de las estudiantes, sobre todo, referidas a su capacidad de observación, transitando desde un estadio de atención difusa que repara en detalles irrelevantes, a saber identificar los acontecimientos significativos que repercuten en el bienestar y aprendizaje de los niños y las niñas de 3 a 6 años.

Cuando entran, en los primeros días están como desconcertadas, abrumadas por tanta actividad, tanto movimiento de los niños, tantas cosas que pasan a la vez en clase... se pierden un poco y están como que no saben a dónde van a acudir... Luego poco a poco, a medida que pasan los días, se van centrando más, van viendo lo que es una rutina, lo que es innovador, lo que es urgente y lo que es importante $[\mathrm{M} 8,1.1$.$] .$

Primero, cuando empiezan a documentar, le echan fotos a todo, sin ninguna selección, es que solo ven, no observan... Luego se dan cuenta que todo no es cuestión de fotografiarlo, que hay que diferenciar lo importante de lo que no lo es [M6,1.1.].

\section{Aprender a escuchar a la infancia}

Por su parte, nueve de las once estudiantes reflejan en sus informes de prácticas, que las competencias profesionales en las que consideran que más han avanzado es en la difícil tarea de escuchar a la infancia. Expresan que es muy fácil de decir, pero extremadamente complejo de hacer porque, como alumnas, han sido socializadas en modelos docentes centrados en la enseñanza, el control y la omnipresencia del profesorado. De esta manera, todas sus representaciones mentales sobre el rol docente las aboca a transmitir contenidos, mantener el control del aula y acaparar el turno de palabra, como funciones irrenunciables para ser una buena docente. Explican que se produce un choque entre sus creencias y el modelo profesional que les ofrecen las maestras del prácticum RIECU, al confrontar su idea de maestra de Educación Infantil, reforzada por las anteriores experiencias de prácticas que han vivido, y la actitud y comportamiento docente de las maestras que trabajan ABP.

Me costó la vida adaptarme al aula porque sin querer me salía... me salía hablar, corregir, dirigir, prohibir, rectificar... a los niños y niñas... les preguntaba algo y antes de que me contestaran ya estaba yo dándole la respuesta correcta. Mi seño me reñía cariñosamente y me decía... déjalos que se expresen y que aprendan de sus errores... todo lo que tú les enseñes estás impidiendo que ellos lo aprendan por sí mismos... [E10,1.2.].

Era increíble cómo hablaban los niños... parecían adultos, con un vocabulario muy elaborado y con mucha seguridad... se expresaban con naturalidad y espontaneidad en público, expresando sus ideas y defendiéndolas cuando diferían de las del resto de compañeros. Cuando proponían los temas del proyecto, la actividad de expertos... era espectacular... Creo que el secreto es que su maestra los escuchaba, los atendía, se los tomaba en serio y no los trataba como niños chiquitos que no pueden hacer casi nada... [E5, 1.2.].

Yo creo que no sabía ser maestra de Infantil antes de conocer a mi tutora de este año. Era más bien una instructora, una máquina de hacer fichas, evaluarlas y dirigir los comportamientos de los niños. Yo pensaba, diseñaba y proponía y ellos ejecutaban mis directrices... realmente no los escuchaba, no los dejaba pensar, crear, argumentar...Quería enseñarles lo que yo sabía y ellos no, pero me olvidaba de todo lo que ellos pueden investigar sin necesidad de que yo les diga la respuesta antes de que puedan descubrirla... [E2,1.2.].

\section{Control emocional para crear un buen clima de aula}

Una constante de todas las estudiantes, y de ocho de las once maestras, así como de dos profesoras universitarias, es constatar el avance en el autocontrol emocional que han experimentado las estudiantes al implicarse en el prácticum RIECU. El inicio en un aula que trabaja por proyectos se percibe por las estudiantes como un reto profesional muy complejo, porque desenvolverse en un ambiente de aprendizaje donde la planificación es muy flexible, donde prevalecen los intereses del alumnado, donde ellos y ellas son los que proponen los temas a investigar, las actividades a desarrollar y los recursos a utilizar, les genera una 
inquietud enorme. No están acostumbradas a desenvolverse en escenarios tan líquidos, tan poco estructurados, tan espontáneos e imprevisibles. Las dudas les asaltan y les generan mucha inseguridad, temiendo no saber qué hacer en el aula si los discentes no proponen nada. Estos temores les generan un miedo inicial que con la ayuda de su tutora profesional se va tornando, aunque no en todos los casos, en una creciente confianza en sí mismas como docentes que pueden liderar un proyecto junto a los niños y niñas.

Yo, el primer día cuando iba para el cole, aparte de no dormir en toda la noche, tenía miedo, pensaba por qué me he metido yo en este lío con lo bien que estuve en clase el año pasado con mi método comercial... Pero ahora no me permitiría haberme perdido esta maravillosa experiencia... [E3, 1.3.]. Cuando entré en la clase y vi a todos los peques tan espabilados, tan autónomos, construyendo su pirámide, con las ideas tan claras... tuve miedo de no poder estar a la altura de las circunstancias. La ayuda de mi tutora ha sido crucial, pero en los primeros días, sobre todo me ayudó mucho a creer un poquito en mí, en que yo también sería capaz de implicarme en el proyecto. [E1, 1.3.]. Estaba nerviosa, inquieta y con miedo de no saber afrontar el desarrollo de un proyecto. Veía a los niños un poco 'forzados' porque al ser el proyecto inducido por las maestras, no los veía muy interesados. No estaban enganchados y se distraían mucho. Mi reto era cómo llamarles la atención, cómo motivarlos. A medida que pasaban los días iba descubriendo 'truquillos'... Lo mejor era escucharlos y respetar sus propuestas... así estaban súper conectados. [E9, 1.3.].

Al principio me enfadaba mucho con ellos cuando no me escuchaban, no respetaban el turno de palabra, no atendían... Elevaba demasiado el tono de mi voz y me sentía desbordada...Mi maestra me enseñó a calmarme, a mantener el control, a tener paciencia y sobre todo a creer en mí, en que soy capaz de hacerlo bien... [E11, 1.3.].

\section{Reflexionar sobre la práctica}

Otra de las competencias más desarrolladas en este proyecto de innovación, en opinión de las maestras, las asesoras y las profesoras, y en menor medida según la visión de las estudiantes, es la reflexión sobre la práctica. Los procesos de investigación-acción colaborativa, desarrollados en las sesiones de reflexión semanales del prácticum RIECU son, según las profesoras universitarias, un momento idóneo para que las estudiantes puedan analizar sus actuaciones en la práctica. Supone un momento privilegiado para la hibridación de la práctica y la teoría, para repensar sus creencias y teorías implícitas en acción, para reajustar su pensamiento práctico. Constatan las contradicciones que se producen entre su decir y hacer como docentes, tomando conciencia de la ralentización que ello supone para su adecuado desarrollo profesional.

Las sesiones reflexivas eran muy formativas porque la estudiante tomaba conciencia de la diferencia que había entre su discurso teórico y su actuación práctica [P1,1.4.].

Eso depende de la alumna que te toque. Yo tuve una alumna que se sabía bien la teoría pero que le costaba mucho la práctica, pero al final lo consiguió. [E7, 1.4.].

Las alumnas no se lo podían creer, pero la documentación pedagógica no engaña, queda constancia a través de imágenes y vídeos... Veían que decían que eran maestras que actuaban como guías y acompañantes, pero en realidad no dejaban hablar a los niños y niñas. Tienen tan incorporado ese modelo docente que lo hacen de manera inconsciente e involuntaria. [A1, 1.4.].

La chica actúa genial en la práctica, tiene madera de maestra, maneja muy bien la clase, pero luego cuando estábamos en la sesión reflexiva, no sabía explicar por qué hacía las cosas. Era como que le salía por generación espontánea, pero sin tener ninguna noción teórica... no sabía ponerle nombre a las cosas que hacía y que hacía muy bien... [M4, 1.4.].

A mí me ponían un poco nerviosa las sesiones de reflexión porque era como todo el mundo evaluándote, mirando con lupa qué haces y cómo lo haces... A veces un poco incómodo, la verdad. Pero es verdad que aprendías... Aprendías lo difícil que es la autocrítica y lo fácil que es decir las cosas, pero lo difícil que es hacerlo [E8, 1.4.].

Las sesiones de reflexión eran momentos interesantes de aprendizaje, aunque mi maestra y yo estábamos reflexionando y comentado lo que hacíamos y lo que pasaba en el aula a todas horas... Pero claro al estar con niños no te podías centrar bien. Cuando comentábamos la documentación, sin los niños, te dabas cuenta de cosas que, sin duda, sin pararte a reflexionar hubieran pasado inadvertidas, al menos para mí. [E3, 1.4.].

\subsection{Fortalezas}

Inyección de moral 
Las maestras, de manera unánime, indican que este año el prácticum RIECU, ha sido una inyección de moral, un 'balón de oxígeno' que les ha motivado y les ha levantado el ánimo en momentos de angustia y apatía provocados por la situación de restricciones derivadas de la pandemia del COVID-19. La situación de aislamiento que han vivido, sin poder compartir anécdotas del día a día en su aula y sin poder salir de sus 'burbujas' ha generado que la llegada de una estudiante de prácticas se viva como una gran ayuda.

Yo, cuando llegó mi alumna, me dio una alegría tremenda, sabía que sería de gran ayuda, tanto para mí, como para mis niños [M7, 2.1.].

Por su parte, las profesoras universitarias y las estudiantes también han expresado que poder vivir el prácticum RIECU ha sido una oportunidad muy valiosa este año, sobre todo, teniendo en cuenta el contexto pandémico que hemos vivido y la imposibilidad de desarrollar el prácticum el curso anterior.

No tenía nada claro que, al final, pudiéramos desarrollar el prácticum innovador, porque el año pasado la pandemia nos lo impidió y no tenía claro que se volviera a repetir la misma situación [P2, 2.1.].

Para mí ha sido un milagro, no me lo creí hasta que estuve en el cole, porque nos decían que se confinaban clases e incluso algún cole... Estaba temerosa porque las cosas cambiaban de un día para otro... [E9, 2.1.].

Al final salió, y fue como un soplo de energía poder verme frente a los peques... Me acordaba del año pasado y me daba una pena tremenda graduarme perdiéndome otro prácticum [E10, 2.1.].

\section{Seducción por la metodología ABP}

Otra de las fortalezas enunciadas por las estudiantes es la seducción que han sentido por la metodología de ABP. Sus expectativas iniciales han sido ampliamente superadas en la mayoría de los casos. Siete de once estudiantes afirman haberse quedado impactadas por los beneficios de esta forma de trabajar.

Al estudiar los proyectos en teoría me llamaban mucho la atención... pero nada que ver con la práctica, con vivirlos y sentirlos en el aula... Es que te envuelven, te metes de lleno en la motivación y la ilusión que existe en el aula...[E1,2.2.].

Estaba pensando todo el día en el proyecto, qué llevar, qué hacer y veía castillos (tema del proyecto) por todos lados. [E3, 2.2.].

Sin embargo, a cuatro estudiantes, no les ha parecido la metodología maravillosa que se ha explicado a nivel teórico, apreciando ciertas discrepancias entre la teoría sobre proyectos aprendida en la facultad y el desarrollo real en las aulas. En algunos casos, también detectan cierto desajuste entre las cualidades que necesita la docente para desarrollar adecuadamente esta metodología y las capacidades personales disponibles.

La verdad que no era como habíamos estudiado en la facultad. Los intereses de los niños se respetaban poco, porque era un proyecto inducido y el tema lo elegían las maestras, que por cierto, todas trabajaban el mismo tema. Los niños no estaban del todo enganchados porque no había surgido de ellos el proyecto... [E6, 2.2.].

A mí, al principio me ha costado bastante... Creo que hay que tener unas cualidades o ser de una forma que yo no soy... Hay que ser muy espontánea, saber improvisar, ser muy flexible... y yo soy muy planificada, muy sistemática, necesito anticipar y tenerlo todo controlado para sentirme segura... Así que me siento más cómoda con las unidades didácticas... [E11, 2.2.].

\subsection{Debilidades y sugerencias de mejora}

\section{Limitaciones derivadas del COVID-19}

Las limitaciones más señaladas por las maestras son las derivadas de las restricciones sanitarias impuestas para evitar el contagio de la pandemia y que hacían que la convivencia en el aula y el desarrollo de los proyectos fuera difícil. Una de las consecuencias más perjudiciales es la limitación en el contacto con otros grupos, así como la eliminación de uno de los pilares básicos del ABP como es la participación familiar, reduciéndola exclusivamente al ámbito digital.

No es lo mismo, las familias, con la cantidad de recursos que aportan a los proyectos solo las podíamos tener en la pantalla. [M12, 3.1.]

Las aportaciones familiares se han diluido mucho... solo como apoyo a los expertos o para grabar vídeos, pero no podían visitarnos en clase. [M5, 3.1.] 
Estábamos todo el día limpiando y algunos materiales no los podíamos usar...era agotador. Tampoco nos podíamos juntar con nuestros compas de nivel, ni de ciclo... Solas, siempre solas, muy penoso... [M8, 3.1.]

\section{Modificación de aspectos organizativos}

Las sugerencias de mejora propuestas por las maestras y las estudiantes hacen referencia a aspectos organizativos como la ampliación de este periodo formativo, más allá de los dos meses establecidos actualmente, así como la ubicación del prácticum en otro momento del curso escolar, coincidiendo con la etapa de adaptación, al considerarse un momento crítico en Educación Infantil.

El prácticum debería durar más... un curso entero. [E1, 3.2.].

Cuando se están acoplando y están cogiéndole el pulso a la clase se tienen que ir. [M12, 3.2.].

El prácticum RIECU estaría genial en el periodo de adaptación. [E6, 3.2.].

\section{Discusión y conclusiones}

La potencialidad del prácticum, como espacio formativo especialmente fértil para la adquisición de competencias profesionales, está ampliamente demostrada en la literatura científica (Mena et al., 2017; Mérida-Serrano et al., 2020; Pérez Gómez, 2012). Sin embargo, estas competencias profesionales pueden enmarcarse en un modelo docente transmisivo, centrado en la reproducción memorística de contenidos o, por el contrario, en responder a un modelo docente innovador que respete el derecho de la infancia a participar en su proceso educativo (Rodríguez-Carrillo et al., 2019). Por esta razón, es necesario realizar una selección de los y las docentes que asumen la tutoría del estudiantado durante su formación inicial, atendiendo a criterios de excelencia educativa, como la formación y la innovación. Este trabajo desvela que las maestras formadas en la metodología de ABP ofrecen modelos profesionales estimulantes que pueden ser imitados por nuestras estudiantes en la fase inicial de su socialización docente, ayudándoles a escuchar a la infancia (Hoyuelos, 2004; Toledo y Sánchez, 2018), a controlar sus emociones para crear un buen clima de aula (Bellver, 2016) y a aprender a reflexionar sobre su práctica con el ánimo de mejorarla (Moss et al., 2005; Ulvik et al., 2018).

Las fortalezas que revelan los datos son la inyección de moral que aportan las estudiantes de prácticas al incluirse en aulas muy limitadas y debilitadas por las restricciones sanitarias adoptadas para evitar el contagio de la pandemia, resultando congruentes con los hallazgos de Romance García et al. (2021) y Renck Jalongo (2021).

Las debilidades y sugerencias de mejora planteadas tienen que ver con la modificación de aspectos organizativos que afectan al Prácticum (extensión y ubicación en el plan de estudios) y que coinciden con las aportaciones de Pérez Gómez (2012), Sousa (2019) y Marín-Cano et al. (2019).

Como conclusión general, debemos indicar que la evaluación del prácticum innovador RIECU revela su potencialidad para incrementar la adquisición de competencias en los y las futuras docentes. Se aprecian los beneficios derivados de la inclusión de estrategias como la selección de docentes atendiendo a criterios de calidad, la pareja educativa, la documentación pedagógica y los procesos de investigación-acción colaborativa. Por ello, como línea futura, se apunta la continuidad en la evaluación de esta experiencia de innovación, así como su transferencia a otros contextos similares para contrastar su pertinencia.

\section{Apoyos}

Experiencia de innovación educativa financiada por el Plan de Innovación y Buenas Prácticas Docentes 2020-2021. Vicerrectorado de Posgrado e Innovación Docente de la Universidad de Córdoba. Referencia: 2020-4-4001. Cuantía: $1500 €$.

\section{Referencias}

Alaçam, N., \& Olgan, R. (2021). Pedagogical documentation in early childhood education: A systematic review of the literature. Elementary Education Online, 20(1), 172-191. https://doi.org/10.17051/ilkonline.2021.01.021

Alsina, A. y Batllori, R. (2015). Hacia una formación del profesorado basada en la integración entre la práctica y la teoría: una experiencia en el Practicum desde el modelo realista. Investigación en la Escuela, 85, 5-18. https://doi.org/10.12795/IE.2015.185.01 
Aragón, L. y Jiménez-Tenorio, N. (2015). El huerto ecológico universitario como herramienta para el estudio de problemas ambientales: una propuesta educativa en el grado de Educación Primaria. Investigar con y para la sociedad, 3, 1839-1844.

Aranguren Peraza, G. (2007). La investigación-acción sistematizadora como estrategia de intervención y formación del docente en su rol de investigador. Revista de Pedagogía, 28(2), 173-195.

Aras, S., \& Tantekin Erden, F. (2019). Documentation panels: supporting young children's self-regulatory and metacognitive abilities. International Joumal of Early Years Education, 28(1), 63-80. https://doi.org/10.1080/09669760.2019.1592743

Aras, S. (2021). Action Research as an Inquiry-Based Teaching Practice Model for Teacher Education Programs. Systemic Practice and Action Research, 34, 153-168. https://doi.org/10.1007/s11213-02009526-9

Arbouet, H. (2010). The Project Approach: A Strategy for Inclusive Classrooms. Young Exceptional, 13(3), 15-27. https://doi.org/10.1177/1096250610364355

Bellver, M. C. (2016). La formación del profesorado en competencias. En I. Carrillo, Democracia y Educación en la formación docente (pp. 87-91). Universitat Central de Catalunya.

Boggino, N. y Rosekrans, K. (2007). Investigación-Acción: reflexión crítica sobre la práctica. Eduforma.

Carr, W. y Kemmis, S. (1988). Teoría crítica de la enseñanza. La investigación acción en la formación del profesorado. Martínez Roca.

Caruso, M. A. (2013). Pareja pedagógica. ¿Cómo trabajar con pareja pedagógica? Escritos en la facultad, 84, $82-83$.

Charles, C. (2009). The Ecology of Hope: Natural Guides to Building a Children and Nature Movement. Journal of Science Education and Technology, 18, 467-475. https://doi.org/10.1007/s10956-009-9193-z

Civarolo, M. M. y Pérez Andrada, M. A. (2013). "1+1= pareja educativa". Nodos y nudos, 4(34), 14-24. https://bit.ly/3Jt84Ep

Coll, R. y Moll, M. C. (2005). La pareja educativa. Una visión práctica. In-fan-cia, 89, $22-29$.

Contreras Domingo, J. (1994). La investigación en la acción: ¿Qué es? Cuadernos de Pedagogía, 224, 8-12.

Elliott, J. (1993). El cambio educativo desde la investigación-acción. Morata.

Hoyuelos, A. (2004). La pareja educativa: un reto cultural. In-fan-cia, 86, 4-10.

TALIS 2018 (2019). Estudio Internacional de la Enseñanza y el Aprendizaje. Informe Español. Ministerio de Educación y Formación Profesional. https://bit.ly/3pDdwwx

Johansson, E. \& Einarsdottir, J. (Eds.) (2017). Values in Early Childhood Education. Citizenship for Tomorrow. Routledge, EECERA.

Kemmis, S. y McTaggart, R. (1992). Cómo planificar la investigación-acción. Laertes.

Korthagen, F. (2010). The relationship between theory and practice in teacher education. En E. Baker, B. McGaw \& P. Peterson (Eds.), International Encyclopedia of Education (Vol. 7) (pp. 669-675). Elsevier.

Luna Arcos, F. y Elorza Ibáñez de Gauna, C. (2009). Educación Infantil: educar en pareja. Cuadernos de Pedagogía, 389, 20-25.

Marín-Cano, M. L., Pava-Bernal, L. R., Burgos-Laitón, S. B., y Gutiérrez-Giraldo, M. M. (2019). La práctica reflexiva del profesor y la relación con el desarrollo profesional en el contexto de la educación superior. Revista Latinoamericana de Estudios Educativos, 15(1), 154-175. https:// bit.ly/3mNdIro

Mauri, T., Clarà, M., Colomina, R., \& Onrubia, J. (2017). Patterns of interaction in the processes of joint reflection by student teachers. Journal of Education for Teaching, 43(4), 427-443. https://doi.org/10.1080/02607476.2017.1296542

McNiff, J. (2002). Action research for professional development. https://bit.ly/3mNanZb

Mena, J., Hennisen, P., \& Loughran, J. (2017). Developing pre-service teachers' professional knowledge of teaching: The influence of mentoring. Teaching and Teacher Education, 66, 47-59. https://doi.org/10.1016/j.tate.2017.03.024

Mérida-Serrano, R. (2005). Una investigación sobre aprendizaje basado en problemas en el marco del Prácticum de Magisterio: una investigación sobre el Prácticum en $2^{\circ}$ de Magisterio de Ed. Infantil. Investigación en la Escuela, 57, 31-46. https://doi.org/10.12795/IE.2005.157.03

Mérida-Serrano, González-Alfaya, E., Olivares-García, M. A., Rodríguez-Carrillo, J., y Muñoz-Moya, M. (2019). Alfabetización profesional de los estudiantes del Grado de Infantil a través de un Prácticum innovador. Revista de Innovación y Buenas Prácticas Docentes, 8(4), 41-57. https://doi.org/10.21071/ripadoc.v8i4.12323

Mérida-Serrano, R., González-Alfaya, M. E., Olivares-García, M. A., Rodríguez-Carrillo, J., y Muñoz-Moya, M. (2020). El Prácticum, un Espacio para la Investigación Transformadora en los Contextos Educativos Infantiles. REICE. Revista Iberoamericana Sobre Calidad, Eficacia y Cambio En Educación, 18(2), 17-34. https://doi.org/10.15366/reice2020.18.2.001 
Moss, P., Dahlberg, G., y Pence, A. (2005). Más allá de la calidad en educación Infantil. Graó.

NAEYC (2002). Early learning standards: Creating the conditions for success. National Association for the Education of Young Children. https://bit.ly/3FKEF6i

OECD (2006). Starting Strong II: Early Childhood Education and Care, Starting Strong. OECD Publishing. https://doi.org/10.1787/9789264035461

Olivares-García, M. A., González-Alfaya, E., y Mérida-Serrano, R. (2016). Reconocernos: Cuando la formación docente inicial y continua se dan la mano. RIECU, Red de Infantil, escuela, centro de profesorado y universidad. En N. Serrano Díaz (Ed.), Innovación docente universitaria en Educación Infantil (pp. 47-65). Octaedro.

Onwuegbuzie, A. J., Dickinson, W. B., Leech, N. L., y Zoran, A. G. (2011). Un marco cualitativo para la recolección y análisis de datos en la investigación basada en grupos focales. Paradigmas, 3, 127-157.

Pérez Gómez, A. I. (2010). Aprender a educar. Nuevos desafíos para la formación de docentes. Revista Interuniversitaria de Formación del Profesorado, 68(24,2), 37-60.

Pérez Gómez, A. I. (2012). Educarse en la era digital. Morata.

PISA 2018 (2019). Programa para la Evaluación Internacional de los Estudiantes. Informe español. Secretaría General Técnica. Centro de Publicaciones. Ministerio de Educación y Formación Profesional.

Pozuelos-Estrada, F., Rodríguez-Miranda F., y Travé-González G. (2012). El enfoque interdisciplinar en la Enseñanza universitaria y el aprendizaje basado en la investigación. Un estudio de caso en el marco de la formación. Revista de Educación, 357, 561-585.

Renck Jalongo, M. (2021). The Efects of COVID-19 on Early Childhood Education and Care: Research and Resources for Children, Families, Teachers, and Teacher Educators. Early Cbildhood Education Journal. https://doi.org/10.1007/s10643-021-01208-y

Ribosa, J. (2020). El docente socioconstructivista: un héroe sin capa. Educar, 56(1), 77-90. https://doi.org/10.5565/rev/educar.1072

Rintakorpi, K., \& Reunamo, J. (2017). Pedagogical documentation and its relation to everyday activities in early years. Early Child Development and Care, 187(11), 1611-1622. https://doi.org/10.1080/03004430.2016.1178637

Rodríguez-Carrillo, J., González-Alfaya, M. E., Mérida-Serrano, R., y Olivares-García, M. A. (2019). Aulas infantiles que trabajan por Proyectos. La interacción social entre iguales como herramienta de aprendizaje. Revista Complutense de Educación, 30(1). https://doi.org/10.5209/RCED.56076

Rodríguez, J., y Cruz, P. (2020). De las competencias básicas a las competencias claves en Educación Infantil. Comparativa y actualización de las competencias en el currículum. Propósitosy Representaciones, 8(1), e366. http://dx.doi.org/10.20511/pyr2020.v8n1.366

Romance García, A. R., Nielsen Rodríguez, A., y Dobado Castañeda, J. C. (2021, 22 de marzo). La pandemia puede asestar un duro golpe a la Educación Infantil. The Conversation. https://bit.ly/3HsI2z8

Shi, Y., Ma, Y., MacLeod, J., \& Yang, H. H. (2019). College students' cognitive learning outcomes in flipped classroom instruction: A meta-analysis of the empirical literature. Journal of Computers in Education, 7, 79103. https://doi.org/10.1007/s40692-019-00142-8

Sousa, J. (2019). Pedagogical documentation: the search for children's voice and agency. European Early Childhood Education Research Journal, 27(3), 371-384. https://doi.org/10.1080/1350293X.2019.1600807

Tejedor, J. (2000). El diseño y los diseños en la evaluación de programas. Revista de Investigación Educativa, 18(2), 319-339. https://revistas.um.es/rie/article/view/121021

Toledo, P., y Sánchez, J. M. (2018). Aprendizaje basado en proyectos. Una experiencia universitaria. Profesorado: Revista de curriculum y formación del profesorado, 22(2), 471-491. https://doi.org/10.30827/profesorado.v22i2.7733

Ulvik, M., Riese, H., \& Roness, D. (2018). Action research-connecting practice and theory. Educational Action Research, 26(2), 273-287. https://doi.org/10.1080/09650792.2017.1323657

UNICEF (2017). Estado Mundial de la Infancia 2017: niños en un mundo digital. Fondo de las Naciones Unidas para la Infancia. UNICEF. https://bit.ly/3sQy5rk

Villela, R., y Torres, C. C. (2015). Modelo de competencias como instrumento de evaluación de la dirección escolar. Revista Iberoamericana de Evaluación Educativa, 8(2), 41-56. https://bit.ly/3pJ0ON0

Zeichner, K., Payne, K. A., \& Brayko, K. (2015). Democratizing teacher education. Journal of Teacher Education, 66(2), 122-135. https://doi.org/10.1177/0022487114560908 